\title{
Análisis de programas nacionales de Extensión Universitaria en América Latina: hacia la Inclusión y la Ciudadanía Cultural
}

\author{
Analysis of national programs of University Outreach in Latin America: \\ towards Inclusion and Cultural Citizenship
}

\section{Análise dos programas de Extensão Universidade Nacional na América Latina: Rumo à Inclusão e Cidadania Cultural}

\author{
Dra. Ahtziri Molina Roldán ${ }^{1}$ \\ Mtro. Aldo Colorado Carvajal \\ Shaila Barradas Santiago \\ Patrick Fowler
}

Palabras clave:

Extensión universitaria

Gestión Cultural

América Latina

Ciudadanía Cultural

Inclusión educativa

Políticas Culturales

\section{Resumen:}

Históricamente, la Extensión Universitaria ha tenido un papel primordial en el desarrollo de los vínculos de la universidad con la sociedad; sin embargo, las nociones que se tienen acerca de ésta función sustantiva son amplias, ambiguas, confusas e incluso contradictorias y por lo tanto, la Extensión no ha podido alcanzar el mismo nivel de consolidación que la otras dos funciones sustantivas de las Instituciones de Educación Superior: la Investigación y la Docencia.

La concepción de las políticas universitarias y tareas a desarrollar desde esta área tienen un carácter singular, pues la Extensión se concibió con un fuerte compromiso social de las Instituciones de Educación Superior con las comunidades que las sustentan. Sin embargo, en la actualidad, la Universidad se ha posicionado como figura hegemónica del conocimiento, dejando poco margen para el aprendizaje de lo que las comunidades aprenden en su vida cotidiana. Por estos motivos consideramos necesario cuestionar los objetivos, las actividades, los actores y el sentido que esta función tiene.

Este artículo busca hacer una revisión comparativa de los elementos y modos de proceder fundamentales de las políticas nacionales de la Extensión Universitaria en cinco países del continente: México, Argentina, Brasil, Colombia y Venezuela. Estas políticas serán analizadas a la luz de los conceptos de ciudadanía cultural y democratización del acceso a los conocimientos universitarios -noción que trasciende la de ampliación de la matrícula-, las cuales se consideran como posibles motores para la construcción de modelos de Extensión Universitaria más incluyentes en la producción y reproducción del conocimiento social generado con y desde las Instituciones de Educación Superior. 
Abstract:

Although University Extension has historically had a prevailingly important role in the formation of the connection between University and society, its conceptualizations are diverse, ambiguous, confusing and at times contradictory; even more so are its practical executions. Therefore, its role has hardly been able to reach the same level of maturity as teaching or investigation.

The perceptions of university policies and of the activities carried out are of a particular in Latin America character because University Extension was conceived as a strong social obligation that higher learning institutions have with the communities that support them. However, many times the practice of Extension, far from sharing information or promoting dialogue, discrediting society's knowledge and positioning the University as a hegemonic figure. For this reason, it is necessary to question the objectives, activities, actors and meaning that this function has.

This paper intends to make a comparative analysis of the national guidelines for University Extension in five Latin American countries (Mexico, Argentina, Brazil, Colombia and Venezuela) and identify the fundamental elements and methods of operation. These policies will be analyzed by way of cultural citizenship and democratization of access to university learning -a notion that transcends that of enlargement of enrollment- which are considered to be possible motivations for the construction of more inclusive and less hegemonic models of University Extension in the production and reproduction of social knowledge generated with and from higher learning institutions.

\section{Keywords:}

University Extension

Cultural Management

Latin America

Cultural citizenship

Inclusion

\section{Palavras chave:}

\section{Extensão Universitária}

Gestão Cultural

América Latina

Cidadania cultural

de inclusão

de Política Cultural

\section{Resumo:}

Historicamente, a Extensão Universitária tem desempenhado um papel importante no desenvolvimento de vínculos entre a universidade ea sociedade; no entanto, eles têm noções sobre o papel substantivo este espaçoso, ambíguo, confuso e até contraditório e, portanto, esta função não tem sido capaz de alcançar o mesmo nível de consolidação como as outras duas funções essenciais das instituições de ensino superior: pesquisa ou ensino.

A concepção de políticas universitárias e as tarefas desta área têm desenvolvido um caráter único que a extensão foi projetada com um forte compromisso social das instituições de ensino superior com as comunidades que lhes dão suporte. No entanto, hoje a universidade tem se posicionado como figura hegemônica de conhecimento, deixando pouco espaço para aprender o que as comunidades aprendem em suas vidas diárias. Por estas razões, consideramos que é necessário questionar as metas, atividades, atores e no sentido de que esta função tem.

Este artigo oferece uma análise comparativa dos elementos e modos de ação das políticas nacionais da Extensão Universitária fundamentais em cinco países do continente do México, Argentina, Brasil, Colômbia e Venezuela. Este artigo baseia-se nas noções de democratização do acesso ao conhecimento universitário, bem como a noção de cidadania cultural, pois ambos são conceitos recorrentes levantadas em casos domésticos. 


\section{Análisis de programas nacionales de Extensión Universitaria en América Latina: Hacia la Inclusión y la Ciudadanía Cultural}

\section{Introducción}

Las universidades juegan un papel central en el proceso de conservación, producción y reproducción del conocimiento de las sociedades en las que están inscritas, así como en la generación de iniciativas que contribuyan a mejorar las formas de vida de la población. Estos cometidos tradicionalmente han sido divididos en tres funciones sustantivas de las Instituciones de Educación Superior (IES): la Docencia, la Investigación y la Extensión Universitaria. Las IES no desarrollan estas funciones con la misma intensidad y dedicación, pues la diversificación institucional no universitaria (educación normal, educación tecnológica) y los nuevos niveles de la educación superior (técnico superior universitario) implican diferentes modelos de entender y hacer la Extensión Universitaria.

Aunado a lo anterior, la Extensión ha tenido poco desarrollo profesional y ha sido objeto de reflexión en mucho menor medida que las otras dos tareas universitarias. Esta situación genera un gran demérito y atención precaria a esta función sustantiva. Aún en este momento en el cual los parámetros de una educación superior exitosa están expresados en términos de calidad y éxito financiero, donde los principales indicadores se refieren a asuntos de Docencia e Investigación, insistimos en la necesidad de reconocer la importancia de las tareas de Extensión y hacer más eficiente la tercera función de la universidad. Lo anterior se puede corroborar en informes de la edu- cación superior, indicadores, etc., donde esta función es contemplada de manera superficial (UNESCO, 2010 ; LÓPEZ SEGRERA, 2011 ; SEP 2013). Más allá de lo supuestamente cuantificable como la calidad en la educación e investigación así como el crecimiento de las matrículas, el trabajo de carácter cualitativo y formativo en torno a las comunidades es vital para que la Universidad comprenda su entorno, así como para colaborar en la construcción de espacios sociales más armónicos y autónomos.

En esta ocasión, haremos un reconocimiento de los postulados de políticas de Extensión Universitaria latinoamericanas. El objetivo es identificar los paradigmas que rigen dichas políticas y el tipo de estrategias para su implementación, además de observar las diferencias y similitudes en los cinco casos analizados, con la finalidad de reconocer las metas planteadas en el trabajo de gestión de estas áreas. El estudio se hace a sabiendas de que resultará un análisis asimétrico por los tamaños de los países tomados como casos de estudio y las políticas empleadas en todos ellos; sin embargo, consideramos que el ejercicio es benéfico para conocer distintos modelos y, con ellos, visiones del tema en diversos puntos del continente.

Para este trabajo se presentan cinco casos del continente los cuales consideramos icónicos y ejemplares de la diversificación que han tenido los sistemas de educación superior y con ello, suponemos, los modelos de Extensión Universitaria. Así se revisan los sistemas de educación superior de Argentina, Brasil, Colombia, México y Venezuela. Estos cinco países, si bien cuentan con sistemas educativos muy diversos, en conjunto concentran casi al $75 \%$ de la matrícula de la región (DIDRIKSSON, 2008). Por lo tanto, consideramos que nos brindarán un crisol importante para la comprensión 
del modo en que han construido tales políticas a nivel continental.

Este estudio pondera dos nociones consideradas como punto de partida y llegada de la Extensión Universitaria. Planteada desde las Reformas de Córdoba, esta función sustantiva fue concebida como un mecanismo de acercar el conocimiento producido en tales instituciones a la población que no tenía acceso a las aulas. Por lo tanto, consideramos que la Extensión Universitaria ha sido una medida para hacer el conocimiento universitario más incluyente y la noción de ciudadanía cultural la vislumbramos como un posible puerto de llegada de los programas de Extensión Universitaria contemporáneos.

\section{La Extensión Universitaria: Tercera Función Sustantiva}

En los últimos años, la matrícula de educación superior en todos los países se ha incrementado, haciendo posible que cada vez sea mayor la población que accede a los conocimientos y la cultura de las IES.

Si bien, cada vez más jóvenes tienen la posibilidad de ingresar a la educación superior, las desigualdades acumuladas históricamente en la región son difíciles de remontar en pocos años: a principios del siglo XXI, menos de la quinta parte de la población en América Latina ha logrado acceder a la educación superior. Si bien se ha ampliado el acceso a ésta, los alcances que aún se tienen siguen siendo menores para muchas poblaciones que históricamente han sido excluidas: indígenas, población rural, mujeres, entre otras.

Aun con los esfuerzos realizados para aumentar el acceso a la educación superior, los resultados en el continente siguen siendo magros; por lo tanto conside- ramos que la Extensión Universitaria tiene un rol importante como medio para hacer llegar a más población los beneficios de la educación superior. Sin embargo, las políticas de las Instituciones de Educación Superior no siempre consideran a esta función como aquella que aporta elementos para el desarrollo de las comunidades y su inclusión en los beneficios que brinda el conocimiento universitario.

Según datos de la CEPAL (2013), de la población mayor de 15 años en América Latina, sólo el $17 \%$ ha alcanzado los 13 años de escolaridad. Ante estas cifras, es evidente que hoy en día, un gran sector de la población queda excluido de los conocimientos y la cultura que se socializan en las universidades e IES. En este sentido es que la extensión tiene mucho que aportar para el desarrollo de nuestras sociedades.

Sin embargo, la problemática mayor que se detecta en cuanto a la Extensión tiene que ver con su definición. Lo que se entiende por Extensión Universitaria varía ampliamente, en especial con la diversidad de tipos de institución y la amplia inserción del sector privado en la Educación Superior. ${ }^{2}$ La particularidad de cada institución, así como de sus objetivos institucionales, modifican las comprensiones de esta función. Es por esto que desde la década de los noventa del siglo pasado, además de las nociones tradicionales de Extensión Universitaria, Difusión Cultural, Comunicación de la Ciencia y Enlace Comunitario, se ha incluido a la Vinculación y, en particular, en relación con el sector productivo por el potencial impacto económico que ésta implicaría. En ocasiones, la Vinculación ha pasado a sustituir a las otras definiciones o tareas de la Extensión en el corazón de las IES. A continuación se realizará una revisión sucinta de las nociones básicas de la Extensión Universitaria. 
Tabla 1. Datos relevantes de los sistemas de educación superior en países seleccionados.

\begin{tabular}{|c|c|c|c|c|}
\hline Pais & Tasa de escolaridad & Matricula pública y privada & \multicolumn{2}{|c|}{ Número de instituciones } \\
\hline $\begin{array}{l}\text { Venezuela }{ }^{3} \\
\text { Población total } \\
27,227,93 \\
(2011)\end{array}$ & $\begin{array}{l}\text { Tasa de cobertura } \\
78 \%(2009)\end{array}$ & $\begin{array}{l}\text { Matricula pública: } 1,581,444(74.72 \%) \\
\text { Matricula privada: } 535,094(25.28 \%) \\
\text { Matricula total: } 2,116,538(2007)\end{array}$ & $\begin{array}{l}48 \text { Universidades } \\
23 \text { Oficiales } \\
25 \text { Privadas } \\
119 \text { Institutos Universitarios }\end{array}$ & $\begin{array}{l}51 \text { Oficiales } \\
68 \text { Privadas } \\
167 \text { IES } \\
(2004)\end{array}$ \\
\hline $\begin{array}{l}\text { México }^{4} \\
\text { Población total } \\
112,336,538 \\
(2010)\end{array}$ & $\begin{array}{l}\text { Tasa de cobertura: } \\
32.8 \%(2012)\end{array}$ & $\begin{array}{l}\text { Matricula pública: } 2,274,311(68.91 \%) \\
\text { Matricula privada: } 1,026,037(31.09 \%) \\
\text { Matricula total } 3,300,348(2013)\end{array}$ & $\begin{array}{l}489 \text { Normal Licenciatura } \\
4198 \text { Licenciatura } \\
2109 \text { Posgrado }\end{array}$ & $\begin{array}{l}438 \text { Federal } \\
1084 \text { Estatal } \\
1478 \text { Privado } \\
6796 \text { IES }\end{array}$ \\
\hline $\begin{array}{l}\text { Argentina }^{5} \\
\text { Población total } \\
40,091,359 \\
(2010)\end{array}$ & $\begin{array}{l}\text { Tasa de cobertura: } \\
75 \%(2010)\end{array}$ & $\begin{array}{l}\text { Matricula pública: } 1,441,845(79.73 \%) \\
\text { Matricula privada: } 366,570(20.27 \%) \\
\text { Matricula total } 1,808,415\end{array}$ & $\begin{array}{l}47 \text { Universidades Nacionales } \\
50 \text { Universidades Privadas } \\
7 \text { Institutos Universitarios } \\
\text { Estatales } \\
14 \text { Institutos Universitarios } \\
\text { Privados }\end{array}$ & $\begin{array}{l}3 \text { Universidad provincial } \\
1 \text { Universidad extranjera } \\
1 \text { Universidad } \\
\text { internacional } \\
133 \text { IES }\end{array}$ \\
\hline $\begin{array}{l}\text { Brasil }^{6} \\
\text { Población total } \\
198,292,000 \\
(2013)\end{array}$ & $\begin{array}{l}\text { Tasa de cobertura: } \\
36 \%(2009)\end{array}$ & $\begin{array}{l}\text { Matricula pública } 1,525,190(25.48 \%) \\
\text { Matricula privada } 4,460,683(74.52 \%) \\
\text { Matrícula total } 5,985,873\end{array}$ & $\begin{array}{l}169 \text { Universidades ( } 83 \text { públicas) } \\
60 \text { Universidades Comunitarias, } \\
\text { Confesionales o Filantrópicas }\end{array}$ & $\begin{array}{l}32 \text { Estatales } \\
46 \text { Federales } \\
5 \text { Municipales } \\
26 \text { Particulares } \\
338 \text { IES }\end{array}$ \\
\hline $\begin{array}{l}\text { Colombia } \\
\text { Población total } \\
47,121,090 \\
(2013)\end{array}$ & $\begin{array}{l}\text { Tasa de cobertura: } \\
45.5 \%(2013)\end{array}$ & $\begin{array}{l}\text { Matricula pública: } 1,012,457(53.96 \%) \\
\text { Matricula privada: } 863,866(46.04 \%) \\
\text { Matricula total } 1,876,323(2011)\end{array}$ & $\begin{array}{l}81 \text { Universidades } \\
119 \text { Instituciones Universitarias/ } \\
\text { Escuelas Tecnológicas }\end{array}$ & $\begin{array}{l}50 \text { Instituciones } \\
\text { Tecnológicas } \\
36 \text { Instituciones Técnicas } \\
\text { Profesionales } \\
286 \text { IES }\end{array}$ \\
\hline
\end{tabular}

\section{Tipos de Extensión Universitaria}

Como se observa, dentro de la Extensión Universitaria existen varios modelos y tipos de actividades que han predominado en distintas instituciones en diferentes periodos, los cuales han impactado en la manera en que se concibe y en cómo se definen las políticas universitarias relacionadas con esta función. Se retoma el trabajo de diversos autores que han elaborado conceptualizaciones sobre esta noción (SÁNCHEZ, 2003 ; SERNA, 2004 ; EJEA Y GARDUÑO, 2014) para establecer definiciones generales sobre las tareas de la tercera función sustantiva.

\section{a. Extensión Universitaria}

Se entiende por Extensión la relación que las instituciones de educación superior tienen con la sociedad no activa en la vida de las mismas (SERNA, 2004, p. 78) y es a través de este espacio que

las universidades... deben contribuir al contacto reflexivo con la realidad, seguida del compromiso para transformarla. La extensión tiene, en potencia, una dimensión educativa de importancia excepcional... a través de la cual la Universidad debe ser una conciencia que no sólo critica, sino que actúa [para] acompañar a 
las personas y grupos humanos en la búsqueda de la respuesta a sus problemas (SERNA, 2004, p. 99-100).

No obstante, pensar en el concepto de Extensión implica pensar en la "integración y transformación mediante la difusión, divulgación, promoción y servicios del conocimiento científico, tecnológico, artístico y humanístico" (ANUIES apud SERNA, 2004, p. 84) que propicie un desarrollo armónico de las sociedades.

\section{b. Difusión Cultural}

Por su parte, la Difusión Cultural tiene como finalidad transmitir a la sociedad los conocimientos resultado de las investigaciones, las concepciones de cultura nacional y universal, las costumbres, entre otras cosas, principalmente de manera unidireccional universidadsociedad, a través de diversos instrumentos como "publicaciones, museos y exposiciones, conferencias, funciones de cine y actividades artísticas" (SERNA, 2004, p. 87) que rara vez se realizan como respuesta a las necesidades e intereses sociales, imponiendo muchas veces visiones del mundo y modos de vida que no son compatibles con sus contextos sociales e históricos.

\section{c. Divulgación o Comunicación de la ciencia}

Según Sánchez (2003, p. 9) "la divulgación de la ciencia es una labor multidisciplinaria cuyo objetivo es comunicar... el conocimiento científico a distintos públicos voluntarios". Con ello, se busca promover el análisis crítico de los hechos sociales para crear conciencia de las situaciones en las que se vive a fin de estimular la participación política de la sociedad para que intervenga en la transformación de sus esquemas de pensamiento, exigiendo mejores condiciones de vida. Los alcances de este modo de extensión han sido magros, pues con frecuencia los lenguajes y espacios de difusión han sido poco apropiados para hacerlo llegar a mayores sectores de la población.

\section{d. Vinculación}

La Vinculación es la relación que se establece entre la universidad y las empresas con el fin de elevar la formación de sus estudiantes al igual que su investigación y así contribuir a la mejor integración a las necesidades sociales de empleo. Basándose en los conceptos de calidad y excelencia educativa, este concepto se apega a las normas del mercado para asegurar el progreso económico del entorno inmediato de la universidad. Sin embargo, muchas veces se cae en una meritocracia radicalizada y una selección cultural acotada que sirve, más bien, para la continuación de las desigualdades sociales (SERNA, 2004, p. 97).

A esta definición dada por Serna habría que agregar que en la actualidad tanto la Asociación Nacional de Universidades e Instituciones de Educación Superior (ANUIES) como muchas IES mexicanas utilizan la noción para referirse a todo tipo de relación establecida con agentes externos a la universidad.

\section{e. Enlace comunitario}

Estas actividades tienen como público específico a los grupos sociales marginados para ofrecer una mejor calidad de vida a través de servicios de salud y de educación, asesoría técnica, entre otros servicios sociales (ANUIES apud SERNA, 2004, p. 84-85).

\section{Inclusión y Ciudadanía Cultural: nocio- nes para comprender la visión contem- poránea de la Extensión Universitaria}

Como se mencionó en la introducción, existen dos conceptos que encontramos presentes en los documentos rectores consultados: la inclusión social 
como una obligación histórica de las Instituciones de Educación Superior, así como la contemporánea construcción que tiene que ver con la ciudadanía cultural. Ambas nociones están presentes en las políticas de educación, pero también, e incluso con mayor énfasis, en las propuestas del sector cultural.

Existen diversas visiones sobre la inclusión, sin embargo una que remite directamente a los trabajos de la tercera función es la propuesta por Chiroleu (2009) quien establece que en las Reformas de Córdoba una de las demandas fue la democratización externa de la Universidad. Lo que esta noción implicaba era ampliar la base social de los asistentes a las aulas de educación superior ${ }^{8}$. A partir de esta demanda es que se gesta la noción de ampliación de la cobertura en la educación superior, la cual buscaba que estudiantes de distintos grupos sociales cursaran estudios universitarios. La meta, según la misma autora, era: "alcanzar una composición interna que refleje la sociedad en su conjunto" (2009, p. 2). No obstante, si bien esta propuesta visualiza el ingreso de los diversos grupos sociales a este nivel educativo no repara en la realidad de que los capitales culturales de los diversos grupos sociales son heterogéneos y por tal, la permanencia y término de los diversos grupos se complica y genera diversas problemáticas.

Es a partir de la identificación de estas problemáticas, y con la finalidad de dar una oportunidad justa a toda la población, que desde la noción de inclusión se asume que la sociedad es heterogénea y por tanto diversa. Es sobre estas bases que se debe de construir la oferta: a partir de las diferencias sociales sin promover su homogeneidad. Este es un término que históricamente ha evolucionado y aunque casi siempre se ha manejado en relación a la matrícula, también ha modificado su comprensión y estrategias de atención a la ciudadanía que no siempre ha tenido acceso al nivel superior.

Así, señala Chiroleu, hoy día se comprende a la inclusión como

procesos más complejos y de una abarcatividad y profundidad mayores. Se apunta a incluir dentro de la universidad una diversidad racial, cultural y sexual semejantes a la que existe en el seno de la sociedad, buscando remediar discriminaciones históricas que han conducido a la situación de desigualdad que han padecido esos grupos. (CHIROLEU, 2009, p. 104)

Aunque actualmente esta definición de inclusión es aún una aspiración, es también un modo de reparar la visión y prácticas parciales y excluyentes que las IES en su mayoría han tenido a lo largo del tiempo. Las medidas prácticas (becas, guarderías etc.) que permitan esta inclusión apenas están en construcción y como procesos de cambio cultural y educativo que son, los resultados que se esperan son a mediano o largo plazo.

Con estas propuestas generadas inicialmente al interior de la vida universitaria se amplía la visión de quién y cómo construye las IES. Por otra parte, se tiene una comprensión más incluyente de lo que se entiende por sociedad y se vuelven más tangibles y cercanas las demandas y situaciones que de otro modo no se habrían visibilizado o comprendido tan bien. Es también aquí donde la noción de intercambio de saberes cobra sentido y se pone al servicio de generar una Universidad más incluyente y sensible al entorno, con lo cual sea posible mejorar la cohesión social y generar mejoras en las condiciones de vida de la población.

Los desafíos son enormes, sin embargo, ya están planteados y en camino de su integración a los núcleos universita- 
rios. La lógica de cambio está en la mesa y en re-elaboración constante.

\section{Hacía la ciudadanía cultural}

La noción de ciudadanía ha acompañado a las sociedades humanas desde los esplendores de Atenas. Se comprende como ciudadanía al conjunto de derechos y deberes por los cuales el individuo está sujeto en relación con la sociedad en la que vive. Es decir, cuando se le otorga al individuo la calidad de miembro de una comunidad organizada. Esta noción está vinculada con un modelo de gobierno democrático.

En la actualidad se identifican tres tipos de ciudadanía: aquella ligada con los derechos políticos, la vinculada con los derechos económicos y la que está relacionada con los derechos culturales. A pesar de que deberían de ser indivisibles en tiempos de libre mercado, no siempre están entrelazadas.

La ciudadanía política es la que se identifica de modo más tradicional, pues es la que confiere el derecho a la residencia, al voto y a la participación política. Mientras que la ciudadanía económica está relacionada con el derecho a trabajar y a prosperar, como el ideal de generar la igualdad, al menos de oportunidades. En tiempos de mercados globales y migraciones laborales, este derecho se presenta en ocasiones de modo independiente a la capacidad de participar en la toma de decisiones políticas de una sociedad.

En tercer lugar mencionamos la ciudadanía cultural, la cual, en su forma básica, provee el derecho del conocimiento y el habla. Además se refiere a la generación de códigos y formas comunes de identificación, creencias y hábitos que permitan la expresión de las representaciones de los colectivos y sus individuos.
La ciudadanía cultural, por ende, tiene tareas importantes en tiempos de globalización e interculturalidad, pues ésta ha de ser la encargada de permitir de modo pacífico la manifestación pacífica y respetuosa de las diversas expresiones culturales que se conjugan en una misma sociedad. Este es el tipo de ciudadanía que exploramos aquí, con la finalidad de identificar cómo se vincula con las IES y los cometidos de la Extensión Universitaria.

Existen diversos modos de comprender y "aplicar" la noción de ciudadanía cultural. Inicialmente, uno de los primeros escollos para la definición de este término es que la noción misma tiene múltiples interpretaciones y puede ser infinitamente incluyente o excluyente. Miller (2011) realiza un recuento de siete posiciones preponderantes sobre el término.

A grandes rasgos se presentan tres posturas: la de Bennet, quien concibe que la ciudadanía cultural es la garantía que tienen las poblaciones de generar un capital artístico y de conocimiento, pero cuyo objetivo es hacerse de competencias para expresarse como individuos y colectivos. Esta postura apunta a la generación de políticas culturales que tengan en mente el derecho a expresarse artísticamente o a disfrutar de tales manifestaciones. Esta visión, si bien cumple objetivos específicos y abre las puertas para la inclusión social, presenta una postura acotada sobre lo que entiende por cultura, pues retoma como base de su definición a las artes como parte central de lo cultural y deja de lado otras expresiones más cotidianas que no necesariamente están atravesadas por la estética -al menos no de modo directo- y resultan tan importantes para la comprensión de lo cultural o más que las propias Bellas Artes.

Rosaldo y Kymlicka están por dar derechos y capacidades similares a los 
desposeídos frente a las mayorías; sin embargo, parten desde sitios opuestos: Rosaldo lo plantea como un modo de resistencia de las minorías que, a través de sus acciones, se construyen un lugar en la sociedad; mientras que Kymlicka propone esta visión como un modo de construir política de estado con la finalidad de incorporar a propios y extraños en un espacio donde la hegemonía está decidida y, al menos económicamente, no será disputada, pero se dará espacio política y culturalmente para participar de la construcción de la nación, siempre y cuando se cumpla de antemano la posibilidad de prosperar económicamente.

Además está la construcción teórica es la desarrollada por Parekh y Hall en el Informe sobre el Futuro de una Gran Bretaña Multiétnica en 2000. Este documento buscaba promover la justicia racial en este país. Los modos que proponen Parekh y Hall para llegar a la realización de este discurso son la suma de los pensamientos enunciados por Bennet, Rosaldo y Kymlicka.

Los autores enunciados hasta ahora reflejan las posibles bondades de una comunidad intercultural puede abonar a las sociedades en transformación, en especial, a aquellas que se consideran desarrolladas y que, como tales, atraen altos números de migrantes a sus suelos. No obstante, el mercado rector de estas mismas sociedades, ve con malos ojos algunos de los usos y costumbres de las comunidades recién llegadas. Tales son los casos que consideran las nociones acuñadas por Amy Chua y el binomio Lewis-Huntington.

Amy Chua en Miller (2011) considera que en estos tiempos el poder económico tiende a concentrarse, mientras que el proceso de democratización aumenta la voz y el poder político de una mayoría frustrada (2003, p. 124 apud MI-
LLER, 2011, p. 67). Considera la autora que la ciudadanía cultural "se trata de un dispositivo que convierte la democracia de libre mercado en una maquina generadora de conflictos étnicos" (2003, p. 6 apud MILLER, 2011, p. 67). Este trabajo se levanta como una advertencia ante la creciente concentración del capital y sus efectos perniciosos en el borde de la desesperación social. Miller considera que la noción de "choque de civilizaciones" de Lewis (1990) y popularizada por Huntington (1993) se utiliza para señalar la diferencia entre el estado laico de occidente y la clara imbricación de la iglesia y el estado de los estados árabes dando como resultado la construcción de lo que se comprende por Estado y de mundo. Por lo tanto, se plantean que los problemas serán principalmente de carácter cultural. Tal posicionamiento cobró fuerza después de los ataques terroristas en Estados Unidos el 11 de septiembre de 2001, donde la prensa y el Estado norteamericano apoyaron esta visión de mundo y de comprensión de lo intercultural y la construcción de derechos ciudadanos en distintos espacios. Sin embargo, no todas las voces líderes de opinión se han sumado a esta postura y entidades como la UNESCO las han refutado directamente. Se plantea que este reduccionismo resta en lugar de sumar en un mundo mucho más complejo de lo que lo plantean.

Si bien la noción de ciudadanía cultural puede tener elementos muy positivos y las primeras concepciones buscan la integración, ya se ve en los últimos dos postulados que no todos están de acuerdo con estos planteamientos y las lecturas de lo cultural son tantas como interpretaciones de este término se proponen.

Como consecuencia, a la hora de pensar la ciudadanía cultural desde las IES es preciso analizar a qué se refieren con ello y cómo buscan implementar las medidas con la finalidad de acuñar qué 
tipo de ciudadano se pretende formar. A fin de cuentas, esta no es una noción objetiva, libre de mancha, y debe de tenerse sumo cuidado en cuáles son las interpretaciones que se dan, cómo se construyen, a qué nociones y a qué intereses responden.

\section{Políticas públicas de la Extensión}

Las definiciones expuestas están en correlación con las políticas de la educación superior, tanto a niveles internacionales como nacionales. En ocasiones, los postulados que se hacen en los distintos niveles son congruentes; en otras más, están sujetos a amplias interpretaciones y muchas otras se resuelven mediante omisión y, por lo tanto, falta de acción en esos sectores.

A continuación se bosquejan los principios generales que la UNESCO ha enunciado sobre la Extensión Universitaria, para después plantear los postulados generales que caracterizan los modelos de Extensión en Venezuela, México, Argentina, Brasil y Colombia. Se eligieron estos países por la diversidad de políticas de Extensión Universitaria que han elaborado las asociaciones o agrupaciones de Instituciones de Educación Superior (IES) en cada país.

En la Conferencia Mundial sobre la Educación Superior de 2009 convocada por la UNESCO se considera como eje central de la declaración final que la educación superior debe de promover el cambio social y el desarrollo de las sociedades. Esto se ha de lograr mediante el fomento de la investigación, la innovación y la creatividad, lo cual es enfatizado en la misión y funciones de la educación superior que tienen que ver con la responsabilidad social de la misma -en especial, la educación superior pública- y donde las tareas de la Extensión son mencionadas en el segundo artículo como una responsabilidad social de las IES para resolver los desafíos mundiales de carácter ambiental, económico, social o cultural. Sin embargo, en lo que respecta a la implementación, la Extensión se desvanece dentro de las acciones que deben llevar a cabo las IES para cumplir con los objetivos propuestos en la declaración (UNESCO, 2010).

\section{México}

El sistema de educación superior de México es uno de los más grandes y complejos en América Latina, tanto por el número de su matrícula como por el número de IES. Sin embargo, de los cinco países analizados, es el de menor cobertura en este nivel educativo: $33 \%$ de la población en edad de estudiar se encuentra matriculado en alguna Institución de Educación Superior del país ${ }^{9}$.

La Asociación Nacional de Universidades e Instituciones de Educación Superior (ANUIES) ha sido en México la principal instancia que ha generado reflexión y organización de las actividades de las IES en México y también la encargada de definir las actividades de Extensión Universitaria.

La ANUIES ha desarrollado una tipificación de las IES con seis perfiles según "su naturaleza funcional académica, las áreas de conocimiento que conforman la oferta académica y los niveles de programas que conforman su oferta" (ANUIES, 2000, p. 13). De este modo, en el Programa Nacional de Extensión Universitaria del año 2000, se caracterizaron los diversos modos de trabajo para el sector con objetivos y estrategias más puntuales.

Un elemento importante de las bases del Programa es que considera distintos tipos de Extensión para cada tipo de institución, de acuerdo a su perfil académico. Esto repercute en que las 
políticas y estrategias que se delineen sean muy generales, ya que se propone que cada IES plantee sus estrategias de acción a partir de un diagnóstico de su quehacer específico.

Según el Programa Nacional de Extensión de los Servicios, Vinculación y Difusión de la Cultura (ANUIES, 2011), último que se elaboró por la Dirección de Extensión de la ANUIES antes de su desaparición en 2013, los fines de ésta son seis: hacer partícipe a la sociedad de los conocimientos de las IES; proporcionar servicios de formación continua; atención y solución de los problemas sociales; la preservación, difusión, enriquecimiento y extensión de las culturas locales y regionales; la retroalimentación de las funciones de Docencia e Investigación; y contribuir al incremento del sector productivo.

Así, el documento establece como políticas fundamentales de la Extensión: la elaboración de reglamentación y normatividad que permita fortalecer las funciones y acciones de la Extensión Universitaria; sumado a esto, la necesidad de generar un presupuesto exclusivo para la Extensión con la finalidad de alcanzar los objetivos planteados por cada IES. Además, resalta el interés en la profesionalización de los responsables de la función.

El Programa Nacional de Extensión de la ANUIES establece que la Extensión debe ser una articulación entre las otras dos funciones sustantivas. La Extensión funciona como un tercer tipo de actividad que pone en relación la Docencia y la Investigación tradicionales.

De esta manera, se delinean reglamentos generales que pueden ser interpretados según el contexto regional de cada institución. Sin embargo, debido precisamente a esta generalidad no hay una conceptualización o definición que permita la concreción de las tareas de la Extensión.

Desde noviembre de 2013, la única oficina que subsiste en el organigrama de la ANUIES es la Dirección General de Vinculación Estratégica. Esta dirección concentra sus actividades en Relaciones Internacionales, Vinculación Interinstitucional y Vinculación Empresarial, dando mayor peso a la relación con el sector productivo (ANUIES, 2012), lo que demuestra la alineación de las políticas educativas nacionales al modelo neoliberal, que va en detrimento de la comprensión de la Extensión Universitaria como un concepto integral y como promotor del desarrollo social. Esta decisión de la Asociación Nacional de Universidades e Instituciones de Educación Superior refleja que las otras áreas de la Extensión no son prioritarias ni necesarias de desarrollar por parte de las IES.

\section{Venezuela}

Venezuela tiene la mayor tasa de cobertura en educación superior entre los países analizados, llegando al $78 \%$. Se caracteriza porque la matrícula es mayoritariamente pública: sólo una cuarta parte se ubica en el sector privado, aunque el número de IES privadas es mayor que el de las públicas ${ }^{10}$.

En Venezuela, el organismo encargado de delinear las políticas de las IES es el Consejo Nacional Universitario, creado en 1946 y que se conforma por el Ministro de Educación, los rectores de las Universidades Nacionales y Privadas y por representantes de profesores y estudiantes de cada universidad.

El Consejo Nacional Universitario creó en 2004 el Núcleo de Autoridades de Extensión (NAEx), aparato responsable de fijar las políticas y coordinar y organizar las acciones de las universida- 
des en el país en las tareas de Extensión. Debido a su reciente creación, este cuerpo todavía se encuentra en proceso de consolidación y fortalecimiento para la coordinación de las actividades de esta función sustantiva en las universidades venezolanas. EI NAEx se considera como un grupo asesor del CNU en materia de Extensión.

Desde el momento de su creación, el NAEx se ha esforzado por establecer una definición clara de Extensión; sin embargo, ha encontrado las mismas dificultades que muchos investigadores sobre esta tarea: existe una variedad de acepciones del término. En la Normativa General para la Extensión, elaborado en 2013, se concibe a la Extensión como "una de las funciones académicas que conjuntamente con la docencia y la investigación, ocupa un lugar preponderante en el desarrollo de las políticas universitarias vinculándose de manera permanente, pertinente y dinámica con la sociedad" (NAEx, 2013-a, p. 1-2).

Las principales actividades a realizar, según los documentos oficiales, son la creación de una estructura universitaria donde se incluyan Direcciones de Extensión, la creación de un presupuesto, la integración de actividades de Extensión en la currícula de estudios y el fomento a la investigación en Extensión (NAEx, 2013a). De esta manera, la Extensión Universitaria se conducirá a formar una parte integral de las universidades venezolanas, integrando a las IES a sus sociedades locales (NAEx, 2013-b).

La Extensión en Venezuela como política universitaria integral todavía se encuentra en una etapa incipiente y se enfrenta a la lucha contra las definiciones predominantes de cultura así como a la ideología política hegemónica del país, pero los propósitos apuntan hacia una Universidad con pertinencia social que responda a las problemáticas comunitarias locales y nacionales.

\section{Argentina}

De los países revisados en este trabajo, Argentina presenta el sistema de educación superior más pequeño, tanto por matrícula como por número de instituciones. Por otro lado, su nivel de cobertura es bastante alta: la educación superior llega al $75 \%$ de la población ${ }^{11}$.

Creado en 1985, el Consejo Interuniversitario Nacional (CIN) es el encargado de aglutinar a las Universidades Nacionales, Institutos Universitarios y Universidades Provinciales argentinas. El $\mathrm{CIN}$ es el encargado de elaborar y coordinar las políticas de desarrollo universitario en las IES que lo integran.

Hace casi cien años, en 1918, fue en Argentina donde se originó la noción de Extensión Universitaria en América Latina. Desde la Reforma de Córdoba, la tercera función en Argentina ha pasado por un largo trayecto de consolidación y formación. En 2008, esa nación conformó la Red Nacional de Extensión Universitaria (REXUNI), la cual actualmente es la encargada de coordinar el fomento y la organización de las actividades de Extensión en el país, aunque ha habido otras organizaciones a lo largo de las décadas que la han precedido.

La Extensión actualmente se entiende como un proceso dinámico donde el conocimiento universitario dialoga con los saberes locales de la sociedad, brindando así una mutua interdependencia y aprendizaje (REXUNI, 2009, p. 1). Se reconoce a la Universidad como generadora de un tipo específico de conocimientos (académico) y que es mediante el intercambio de saberes que se puede posicionar a la Universidad como un actor social que incide en los problemas sociales para crear nuevas y mejores opor- 
tunidades de desarrollo. El concepto de Extensión va más allá de la ampliación de la oferta educativa o del acceso a la educación; sin embargo, tampoco llega a considerar, en gran medida, los derechos culturales de la sociedad.

En Argentina, la Extensión tiene el encargo de responder a los problemas nacionales de diversa índole. Sus principales ejes de trabajo tratan de los derechos humanos de primera generación, la transformación económica, la cuestión ambiental y democratización de la comunicación social.

El "extensionismo" argentino se presenta como uno de los más academizados de la región y muchas universidades en el país lo contemplan dentro de los planes de estudio como una actividad académica a desarrollar, incluyendo a todos los agentes académicos que trabajan en él. Además, es considerada una práctica profesional: se realizan cursos y diplomados a nivel nacional de capacitación para personal extensionista (REXUNI, 2013).

\section{Brasil}

Brasil tiene la mayor matrícula de los países estudiados, con casi seis millones de estudiantes. Sin embargo, su tasa de cobertura es una de las más bajas de la región, alcanzando solamente a $36 \%$ de la población en edad escolar. A diferencia de los otros cuatro países aquí analizados, la mayoría -casi el 75\%-de la matrícula brasileña está inscrita en IES privadas ${ }^{12}$

La Universidad en Brasil surgió tardíamente -en la primera mitad del siglo XX-pero gracias a esto siempre ha existido un ambiente de necesidad de Extensión en el marco político del país. Desde sus inicios, y antes de conceptualizarse formalmente, las universidades brasileñas ya reconocían la necesidad del diálogo de saberes entre la academia y la sociedad. En 2008, la Extensión fue llevada a nivel de política educativa nacional, cuando el Programa de Extensão Universitária (PROEXT) fue creado por decreto presidencial dentro del ámbito del Ministério de Educação, lo cual convierte al Programa no sólo en política educativa, sino en política pública de Estado. Esto, además de una historia de investigación sobre las actividades de Extensión, brinda a Brasil un alto nivel de maduración en este ámbito.

En este país, la manera de crear vínculos entre la Universidad y la sociedad en general es mediante actividades de Extensión Universitaria, de modo que esta función está volcada hacia la resolución de los problemas sociales locales de cada universidad. Las directrices de la Extensión Universitaria mueven a ésta hacia la interacción dialógica, la interdisciplina, una indisociabilidad entre las funciones universitarias, el impacto en la formación estudiantil y la transformación social (BRASIL, 2012).

Al hablar de la indisociabilidad entre las actividades sustantivas de la Universidad, se considera que la legitimidad de las universidades sólo se cumplirá cuando las actividades de Extensión pasen a ser una parte integral de la Investigación y la Docencia, lo cual va más allá de la difusión de conocimiento, la prestación de servicios o la difusión cultural (BRASIL, 2006). De esta manera, se retira el concepto de "tres funciones universitarias" a cambio de la incorporación total de la Extensión dentro de las demás actividades. La Extensión se define, entonces, como la actividad académica que da rumbo a la Universidad y que contribuye significativamente para cambiar a la sociedad (BRASIL, 2001, p. 1). Se reconoce la importancia de los retos específicos dentro de una región y las universidades públicas están encargadas de incidir en la creación de políticas públicas para enfrentar las demandas de su población local. 
En Brasil, el intercambio de saberes es muy importante para las actividades de Extensión. Según el Plano Nacional de Extensão Universitária, la Extensión produce conocimiento "a cambio de saberes sistematizados, académicos y populares, teniendo como consecuencia la democratización del conocimiento, la participación efectiva de la comunidad en las actividades de la Universidad y una producción resultante de la confrontación con la realidad." (BRASIL, 2001, p. 4). De esta manera, la Extensión es concebida como "filosofía, acción vinculada, política, estrategia democratizante, metodología” (BRASIL, 2001, p. 5).

\section{Colombia}

Basado en las estadísticas retomadas, el sistema de educación superior en Colombia presenta un punto intermedio entre los países estudiados aquí. El número total de Instituciones de Educación Superior se encuentra en el medio del rango y su tasa de cobertura es de $45.5 \%$. Su matrícula se divide casi a la mitad entre universidades públicas y privadas ( $54 \%$ pública y $46 \%$ privada). ${ }^{13}$

En 1957 se crea la Asociación Colombiana de Universidades (ASCUN) como un medio de interlocución de las Universidades con el Estado y la sociedad colombiana. El ASCUN abarca el $30 \%$ de las IES del país y está dirigido por el Consejo Nacional de Rectores.

En 2013, fue elaborado el documento Políticas culturales para la educación superior en Colombia en el que se establecen las bases para el ejercicio de la gestión cultural desde las IES, con el propósito de generar una política común en el quehacer de las actividades culturales en la sociedad y de esta forma contribuir al desarrollo social de Colombia.
Esta propuesta elaborada a lo largo de un considerable tiempo y con diversos actores involucrados, se basa tanto en documentos internacionales como las Declaraciones Mundiales de la UNESCO, como en la legislación nacional colombiana, además de documentos universitarios. Esta combinación de perspectivas sobre la cultura da como resultado una concepción de ésta como un elemento trascendental en el desarrollo social y la posibilidad de un cambio profundo al incluir nociones como derechos culturales, sustentabilidad, patrimonio cultural e inclusión, entre otros.

De esta forma, del sentido que se le da a la Extensión Universitaria desde la perspectiva de la gestión cultural universitaria, se entiende como:

una actividad mediante la cual la universidad trasciende su propio espacio físico, se abre al mundo y se integra e interactúa con la sociedad de la que forma parte. Esto hace de la cultura una dimensión ineludible en los procesos de extensión, pues permite dar cuenta de los imaginarios, las expectativas, los contextos y sus particularidades, las vocaciones regionales y locales, y las formas como se establecen las relaciones entre los sujetos, como elementos claves para la apropiación de conocimientos, desarrollos científicos y nuevas tecnologías, entre otros aspectos que encarnan la misión de la extensión o proyección social en las instituciones de educación superior. (JARAMILLO ET AL., 2013, p. 81)

De esa forma, la Extensión rebasa la divulgación del conocimiento científico para proponer un proyecto de construcción de vida cultural de la que se desprende la noción de ciudadanía intercultural, en el cual la Universidad tiene un papel proactivo en la sociedad. Los ejes de trabajo considerados por las universidades colombianas se enfocan en los derechos 
culturales, la construcción de ciudadanía cultural, el desarrollo humano sostenible y pertinente, el diálogo de saberes y la inserción de la cultura en la vida académica. Estos ejes se organizan según lineamientos como la dimensión sustantiva de la educación superior; la formación cultural y su contribución a la formación universitaria, la ciudadana intercultural y de públicos; la creación y el diálogo intercultural como expresiones de los derechos culturales; los patrimonios y memorias culturales activos de la sociedad; nueva gestión del conocimiento: la ciencia, la tecnología y la innovación en clave de cultura; Universidad, cultura y territorio; Universidad y gestión cultural; comunicación pública y circulación de conocimientos, expresiones, bienes y servicios culturales; y la universidad como actor político-cultural.

La iniciativa de Colombia de convertir la Extensión en parte de una política cultural nacional no es exclusiva a esta nación, también se ha presentado en los casos de Brasil y de Bolivia.

\section{Consideraciones finales}

El recuento aquí presentado nos brinda la oportunidad de analizar los objetivos y alcances de las diferentes políticas de Extensión Universitaria de la región. Los casos se han presentado de acuerdo a los niveles de complejidad y abstracción que estos presentan. Se considera que los casos iniciales prestan más atención a asuntos operativos y están más centrados en la organización intra-universitaria mientras que los casos hacia el final presentan más proyección social.

Para comenzar, cabe mencionar que todos los países han buscado consenso a nivel nacional para determinar los roles que tienen o aspiran a tener las entidades de Extensión Universitaria en cada país. Es notorio que en la primera déca- da de este siglo todos los países hicieron reuniones nacionales (incluso previo a la reunión de 2009 de la UNESCO) para fortalecer esta función. Como resultado de estas reuniones en Brasil y Colombia la Extensión Universitaria toma el papel de políticas educativas nacionales reconocidas constitucionalmente como herramienta fundamental del trabajo universitario. Además de que en el caso colombiano la tercera función se considera un agente de la agenda política nacional y participante en la elaboración de políticas culturales y científicas de la nación.

Sin embargo, los derroteros y alcances que han perseguido para esta función difieren ampliamente. Mientras el caso mexicano de modo fáctico -mediante la eliminación de la oficina de Extensión de la ANUIES- ha decidido concentrar los esfuerzos de esta función acercándola al sector productivo y buscando en este generar una fuente de financiamiento para las IES a través de la figura de la vinculación, en otros países consideran la Extensión Universitaria desde su visión integral e integradora de las otras funciones universitarias, en relación tanto al conocimiento como a la sociedad que les da sustento e identidad.

Argentina, Brasil y sobre todo Colombia han dotado a esta función de capacidades para incidir en el desarrollo, un agente con presencia en políticas públicas y promotor del mejoramiento de la vida social. A través de postulados como el intercambio de los saberes con las comunidades, se considera que la Universidad aprende de su entorno y participa del mejoramiento de tales comunidades y se mejora a sí misma. Del mismo modo, el modelo argentino apunta a que de esta forma se puede generar mayor inclusión (CHIROLEU, 2009), sobre todo de aquellas poblaciones que difícilmente tendrían acceso a los estudios universitarios por su condición socio-económica, lugar de 
residencia o alguna otra situación; con lo cual valoran el diálogo de saberes como herramienta para relacionarse fuera del ámbito universitario.

A su vez, los modelos brasileño y colombiano consideran que la Extensión es una herramienta para promover la inclusión, abriendo paso a la posibilidad de hacer el trabajo de la tercera función de modo integral. De esta manera, la inclusión es una posible vía para la democratización del conocimiento y una fuente importante de la construcción de la ciudadanía.

Por su parte, Argentina y Colombia han establecido políticas y modos para la capacitación de los extensionistas; con lo cual se refuerza de modo práctico el papel de la tercera función a la vez que se acerca a tener los mismos roles que la Investigación y la Docencia. La diferencia fundamental entre los modelos de capacitación de estas dos naciones estriba en el hecho de que; mientras Argentina ha buscado hacer de cada agente universitario un extensionista, Colombia ha buscado capacitar en gestión cultural al sector propiamente destinado a esta tarea y generar cuerpos especializados en esta función.

Un paso más que dan Brasil y, sobre todo, Colombia es la posibilidad de tener en la Extensión Universitaria una herramienta capaz de construir nuevos modos de convivencia al interior y exterior de la vida universitaria, mediante la consideración de la promoción de la ciudadanía intercultural como meta. Aunque la aplicación de este término varía por países, pues Brasil la menciona pero no alcanza a definir su acepción en los documentos mientras que Colombia reconoce en este paradigma que la Extensión se ve favorecida como agente política de cambio al ser capaz de

propiciar el ejercicio de los derechos humanos y culturales y una partici- pación efectiva en la vida cultural, como fundamento para una sociedad más respetuosa de la diversidad, más dialógica y capaz de construir desde su memoria cultural y desde sus prácticas culturales, los referentes que nos permitan ser, cada día, una mejor sociedad (JARAMILLO ET. AL., 2013, p. 59).

Por lo tanto, la noción utilizada por estos dos países apunta a mirar la ciudadanía cultural como una vía para generar un mejor modo de vida donde la aceptación de la diversidad es considera eje central en la construcción y modificación de la vida de las comunidades.

Las conceptualizaciones abstractas como estas, operacionalizadas mediante las políticas universitarias, dan paso a que la Extensión construya un universo propio y un área de influencia mayor, haciendo que la presencia de las tareas de las IES sea más contundente de modo cotidiano como agente de la vida social y pública.

Como podemos ver, no existe una sola visión sobre lo que la Extensión Universitaria implica en América Latina. Son claros los esfuerzos que se están realizando en los diversos países -sobre todo en Colombia, Argentina y Brasil- por comprender mejor el contexto social, político y económico en el que las IES están inmersas y procurar un nuevo establecimiento de relaciones con el entorno.

Desde el discurso, hay ahora una comprensión mayor de que la Extensión debe de responder al contexto y no a los parámetros internacionales de educación superior, pues, finalmente, la Universidad se debe es a estas poblaciones. Sin embargo, aún es necesario trabajar en su implementación en la realidad sin dejar de reconocer que las bases están planteadas $y$, con ello, hay rutas que seguir. 


\section{Bibliografia}

ASOCIACIÓN NACIONAL DE UNIVERSIDADES E INSTITUCIONES DE EDUCACIÓN SUPERIOR (ANUIES). Programa Nacional de Extensión de la Cultura y los Servicios. XXX Sesión Ordinaria de la Asamblea General de ANUIES. México: ANUIES, 2000.

ASOCIACIÓN NACIONAL DE UNIVERSIDADES E INSTITUCIONES DE EDUCACIÓN SUPERIOR (ANUIES). Programa Nacional de Extensión de la Cultura y los Servicios. XXX Sesión Ordinaria de la Asamblea General de ANUIES. México: ANUIES, 2011.

ASOCIACIÓN NACIONAL DE UNIVERSIDADES E INSTITUCIONES DE EDUCACIÓN SUPERIOR (ANUIES). Plan de Desarrollo hacia el 2016. Hacia la proyección de la vinculación de las IES para la innovación orientada al desarrollo sustentable de las regiones de México. México: ANUIES, 2012.

BARROS, Enrique; VALDÉS, Horacio et. al. Manifiesto Liminar de la Reforma Universitaria del 21 de junio de 1918. Argentina: Universidad de Córdoba. Disponible http://www.unc.edu.ar/institucional/historia/reforma/manifiesto Consultado el 28 de febrero de 2014.

BRASIL. MINISTÉRIO DA EDUCAÇÃO. PROGRAMA DE EXTENSÃO UNIVERSITÁRIA. Plano Nacional de Extensão Universitária Edição Atualizada. Brasil: PROEXT, 2001.

BRASIL. MINISTÉRIO DA EDUCAÇÃO. PROGRAMA DE EXTENSÃO UNIVERSITÁRIA. Indissociabilidade ensino-pesquisa-extensão e a flexibilização curricular: uma visão da extensão. Brasil: UFRGS / MEC / SESu, 2006.

BRASIL. MINISTÉRIO DA EDUCAÇÃO. PROGRAMA DE EXTENSÃO UNIVERSITÁRIA. Política nacional de extensão universitária. Brasil: PROEXT, 2012.

COMISIÓN ECONÓMICA PARA AMÉRICA LATINA Y EL CARIBE. Unidad de Estadísticas Sociales, sobre la base de tabulaciones especiales de las encuestas de hogares de los respectivos países. División de Estadísticas, 2013. Disponible http://estadisticas.cepal.org/cepalstat/WEB_CEPALSTAT/estadisticas Indicadores.asp?idioma $=e$ Consultado el 28 de noviembre de 2013.

CHIROLEU, Adriana. La democratización del acceso a la Universidad: de la ampliación de opor- tunidades a la inclusión. In: CHIROLEU, Adriana; MARQUINA, Mónica. A 90 años de la Reforma Universitaria: memorias del pasado y sentidos del presente. Argentina: Universidad Nacional de General Sarmiento, 2009. p. 99-116.

DIDRIKSSON, Axel. Contexto global y regional de la educación superior en América Latina y el Caribe. In: GAZZOLA, Ana Lucia; DIDRIKSSON, Axel (editores). Tendencias de la Educación Superior en América Latina y el Caribe. Colombia: IESALC-UNESCO, 2008.

GARDUÑO, Bianca ; EJEA, Tomás. La tercera función sustantiva de las Instituciones de Educación Superior Privadas en México. Una propuesta metodológica para su análisis, (En prensa), 2014.

JARAMILLO GONZÁLEZ, María Adelaida ; MUÑOZ ÑÁÑEZ, Paloma ; MEJÍA ARGÜELLO, Luis Álvaro ; MIRA FERNÁNDEZ, Verónica ; MARTINELL SEMPERE, Alfons. Políticas culturales para la educación superior en Colombia. Medellín, Colombia: Universidad de Antioquia, 2013.

LÓPEZ SEGRERA, Francisco. La educación superior en el mundo y en América Latina y el Caribe: principales tendencias In: DOS SANTOS, Theotonio. América Latina y el Caribe: escenarios posibles y políticas sociales. Montevideo, Uruguay: FLACSO-UNESCO, 2011, p. 204-255.

MILLER, Toby. Cultural Citizenship. MATRIZes, Año 4 no2. São Paulo, Brasil, 2011, p. 57-74.

NÚCLEO DE AUTORIDADES DE EXTENSIÓN DE LAS INSTITUCIONES DE EDUCACIÓN UNIVERSITARIA. Normativa general de Extensión para las Instituciones de Educación Universitaria debidamente autorizadas por el CNU. Venezuela: NAEx, 2013-a.

NÚCLEO DE AUTORIDADES DE EXTENSIÓN DE LAS INSTITUCIONES DE EDUCACIÓN UNIVERSITARIA. Plan estratégico nacional de Extensión Universitaria (2013-2018). Venezuela: NAEx, 2013-b.

ORGANIZACIÓN DE LAS NACIONES UNIDAS PARA LA EDUCACIÓN, LA CIENCIA Y LA CULTURA (UNESCO). Communiqué. Conferencia Mundial sobre la Educación Superior - 2009: La nueva dinámica de la educación superior y la investigación para el cambio social y el desarrollo. París, Francia: UNESCO, 2010.

PROGRAMA PARA EL MEJORAMIENTO DEL PROFESORADO (PROMEP). Filosofía de Cali- 
dad. México: PROMEP-SEP, 2013. Disponible en http://promep.sep.gob.mx/ Consultado el 17 de febrero de 2014

RED NACIONAL DE EXTENSIÓN UNIVERSITARIA. Conclusiones del III Congreso Nacional de Extensión: La integración, extensión, docencia e investigación. Desafíos para el desarrollo social. 20-22 de julio. Argentina: Universidad Nacional del Litoral, 2009.

RED NACIONAL DE EXTENSIÓN UNIVERSITARIA. Capacitación en Extensión Universitaria. Argentina: REXUNI, 2013.

SÁNCHEZ MORA, Ana María ; SÁNCHEZ MORA, Carmen. Glosario de términos relacionados con la divulgación: una propuesta. El Muégano Divulgador, México, n.21, 2003, p. 9.

SECRETARÍA DE EDUCACIÓN PÚBLICA (SEP). Programa Sectorial de Educación 2013-2018. México: Secretaría de Educación Pública, 2013.

SERNAALCÁNTARA, Gonzalo. Modelos de extensión universitaria en México. Revista de la Educación Superior, México, vol. XXXIII, n. 131, 2004, p. 77-103. Disponible en http://publicaciones.anuies. $\mathrm{mx} /$ revista/131/2/1/es/modelos-de-extension-universitaria-en-mexico. Consultado el 12 de diciembre de 2013.

\section{Recebido em 26/11/2014 Aprovado em 10/02/2015}

1 Grupo vinculado ao Seminario de Investigación en Gestión Cultural Centro de Estudios, Creación y Documentación de las Artes, Universidad Veracruzana, México. Contatos: ahtziri@gmail.com , aldococa@hotmail. com , shailabarradas@gmail.com e patrickmfowler@ gmail.com

2 Ejea y Garduño (2014) señalan que el día de hoy en México dos terceras partes de la Educación Superior está en manos de la Iniciativa Privada, lo cual modifica drásticamente el panorama del sector y sus cometidos.

3 Ministerio del poder popular para la educación. La revolución Bolivariana en la educación superior. 10 años de logros. [en línea] junio 2009 [fecha de consulta: 24/09/2014] disponible en: http://venezuela-us.org/es/wp-content/ uploads/2009/09/10-anos-de-logros-junio-2009.pdf Instituto Nacional de Estadísticas. Resultados básicos. Censo 2011. [en línea] Caracas. Agosto 2012 [fecha de consulta: 24/09/2014] disponible en:

http://www.ine.gov.ve/documentos/Demografia/ CensodePoblacionyVivienda/pdf/ResultadosBasicosCenso2011.pdf

4 Secretaría de Educación Pública. Principales Cifras del Sistema Educativo Nacional 2013-2014. [en línea] primera edición. México D.F. 2013 [fecha de consulta: 24/09/2014] disponible en: http://fs.planeacion.sep.gob. mx/estadistica_e_indicadores/principales_cifras/principales_cifras_2012_2013_bolsillo.pdf

Instituto Nacional de Estadística y Geografía. Conociendo México [en línea] Aguascalientes, Ags. Septiembre 2012. [fecha de consulta: $24 / 09 / 2014$ ] disponible en: http://www.inegi.org.mx/prod_serv/contenidos/espanol/ bvinegi/productos/integracion/pais/mexcon/folleto_nacional_pliegos_baja.pdf

5 Acerca del sistema universitario. Recuperado el: 24 de septiembre de 2014, del sitio web del Ministerio de Educación http://portal.educacion.gov.ar/universidad/ sistema-universitario/acerca-del-sistema-universitario/ Ministerio de Educación. Presidencia de la Nación. Anuario 2011 Estadísticas Universitarias, Argentina [en línea] Buenos Aires, Argentina [fecha de consulta: 24/09/2014] disponible en:

http://informacionpresupuestaria.siu.edu.ar/DocumentosSPU/Anuario $\% 20 \mathrm{de} \% 20$ Estad $\%$ C3\%ADsticas $\% 20$ Universitarias\%20-\%20Argentina\%202011.pdf ISSN 1850-7514

6 La educación superior en el Mercosur: Argentina, Brasil, Paraguay y Uruguay hoy. [PDF] 1ra edición. Buenos Aires, Argentina: Editorial Biblos, 2012 [fecha de consulta: 24/09/2014] disponible en:

file:///C:/Users/PAVILION/Downloads/Version_diciembre_ del_libro_la_Educacion_Superior_en_el_Mercosur.pdf

7 Ministerio de Educación Nacional. ESTADÍSTICAS DE EDUCACIÓN SUPERIOR [PDF] 31 de diciembre de 2013 [fecha de consulta: 24/09/2014] disponible en: http://www.mineducacion.gov.co/sistemasdeinformacion/1735/articles-212350_Estadisticas_de_Educacion_ Superior_.pdf

Banco de la República, [en línea] http://www.banrep. gov.co/es/poblacion [fecha de consulta: 24/09/2014]

8 Véase el Manifiesto Liminar de la Reforma Universitaria del 21 de junio de 1918.

9 Véase Tabla 1.

10 Véase Tabla 1

11 Véase Tabla 1.

12 Véase Tabla 1.

13 Véase Tabla 1 